\title{
NeuronUnit: A package for data-driven validation of neuron models using SciUnit
}

\author{
Richard C. Gerkin ${ }^{1, *}$, Justas Birgiolas ${ }^{1, *}$, Russell J. Jarvis ${ }^{1}$, Cyrus Omar ${ }^{3}$, \\ Sharon M. Crook ${ }^{1,2}$ \\ ${ }^{1}$ School of Life Sciences, Arizona State University, Tempe, AZ, USA \\ ${ }^{2}$ School of Mathematical and Statistical Sciences, Arizona State University, Tempe, \\ AZ, USA \\ ${ }^{3}$ Dept. of Computer Science, Univ. of Chicago, Chicago, IL, USA \\ Correspondence*: \\ Richard C. Gerkin \\ P.O. Box 874501, Tempe, AZ USA 85287-4501, rgerkin@asu.edu
}

\section{Not peer-reviewed}

\begin{abstract}
Validating a quantitative scientific model requires comparing its predictions against many experimental observations, ideally from many labs, using transparent, robust, statistical comparisons. Unfortunately, in rapidly-growing fields like neuroscience, this is becoming increasingly untenable, even for the most conscientious scientists. Thus the merits and limitations of existing models, or whether a new model is an improvement on the state-of-the-art, is often unclear.

Software engineers seeking to verify, validate and contribute to a complex software project rely on suites of simple executable tests, called "unit tests". Drawing inspiration from this practice, we previously developed SciUnit, an easy-to-use framework for developing data-driven "model validation tests" - executable functions, here written in Python. Each such test generates and statistically validates predictions from a model against one relevant feature of empirical data to produce a score indicating agreement between the model and the data. Suites of such validation tests can be used to clearly identify the merits and limitations of existing models and developmental progress on new models.

Here we describe NeuronUnit, a library that builds upon SciUnit and integrates with several existing neuroinformatics resources to support the validation of single-neuron models using data gathered by neurophysiologists and neuroanatomists. NeuronUnit integrates with existing technologies like Jupyter, Pandas, NeuroML and resources such as NeuroElectro, The Allen Institute, and The Human Brain Project in order to make neuron model validation as easy as possible for computational neuroscientists.
\end{abstract}

Keywords: Neuroinformatics Simulation Electrophysiology Software Modeling Validation

\section{INTRODUCTION}

\subsection{THE PROBLEM: WHAT DO MODELS DO AND HOW WELL DO THEY DO IT?}

Neuroscientists construct quantitative models to explain experimental observations of ion channels, neurons, circuits, brain regions and behavior. A model can be characterized by its scope: the set of observable 
quantities that it can generate predictions about, and by its validity: the extent to which its predictions agree with available experimental observations of those quantities.

Today, neuroscientists typically contribute a model to the research community by submitting a paper describing the model's structure and scope, arguing for its novelty, and containing a very limited selection of tables and figures demonstrating its validity. Those tasked with reviewing the paper are responsible for evaluating these claims, discovering competing models and relevant data the paper did not adequately consider, and ensuring that goodness-of-fit was measured in a statistically sound manner, drawing on their knowledge of prior literature. Often, there are no means for verifying even the basic results 1 . And once a peer-reviewed modeling paper is published, it is frozen and cited as-is in perpetuity. Because publications are the sole vehicle for knowledge about model scope and validity, investigators must rely on an encyclopedic knowledge of the literature to answer summary questions like the following:

- Which models are capable of predicting the measurable quantities I am interested in?

- What are the best practices for evaluating the goodness-of-fit between these models and data?

- How well do these models perform, as judged by these metrics, given currently available data?

- What other measurable quantities can and can't these models predict?

- What observations have not yet been adequately explained by any available model?

In some fields, like neuroscience, where the number of relevant publications being generated every year is growing rapidly ${ }^{2}$, these questions can be difficult for even conscientious senior scientists to answer comprehensively. For new computational neuroscientists, this represents a serious barrier to entry.

\subsection{THE SOLUTION: UNIT TESTING FOR MODELS}

Professional software developers face similar issues ${ }^{3}$. They must understand the scope of each component of a complex software project and verify that each component achieves desired input/output behavior. But software developers do not verify components by simply documenting a few interesting inputs and corresponding outputs and then presenting them to other developers for one-time review leading to an archived document. Rather, they typically follow a test-driven development methodology by creating a suite of executable unit tests that serve to specify each component's scope and verify its implementation on an ongoing basis as it is being developed and modified 4 . Each test individually checks that a small portion of the program meets a single correctness criterion. For example, a unit test might verify that one function within the program correctly parses inputs of a certain type. Collectively, the test results serve as a summary of the project as it progresses through its development cycle. Developers can determine which features are unimplemented or buggy by examining the set of failed tests, and progress can be measured in terms of how many tests the program passes over time. This methodology is widely adopted in practice ${ }^{4-6}$.

Test-driven methodologies have started to see success in neuroscience as well, in the form of modeling competitions. During these competitions, competitors develop and parameterize models based on publicly-available training data and submit them to a central server. There, submitted models are provided hidden testing data which they must use to produce predictions. These predictions are validated using publicly available criteria to produce summaries of the relative merits of different models, just as a test suite summarizes the state of a software project. Such competitions continue to drive important advances and improve scientists' understanding of their fields. For example, the DREAM olfaction challenge asked participants to build models predicting the odor qualities of molecules, and ranked models based on their ability to predict the odors of novel molecules ${ }^{7}$. The winning model in this challenge currently represents the state-of-the-art in structure-odor predictions. More relevant for neurophysiology and neuroanatomy, the quantitative single neuron modeling competition (QSNMC) $)^{[8}$ investigated the complexity-accuracy 


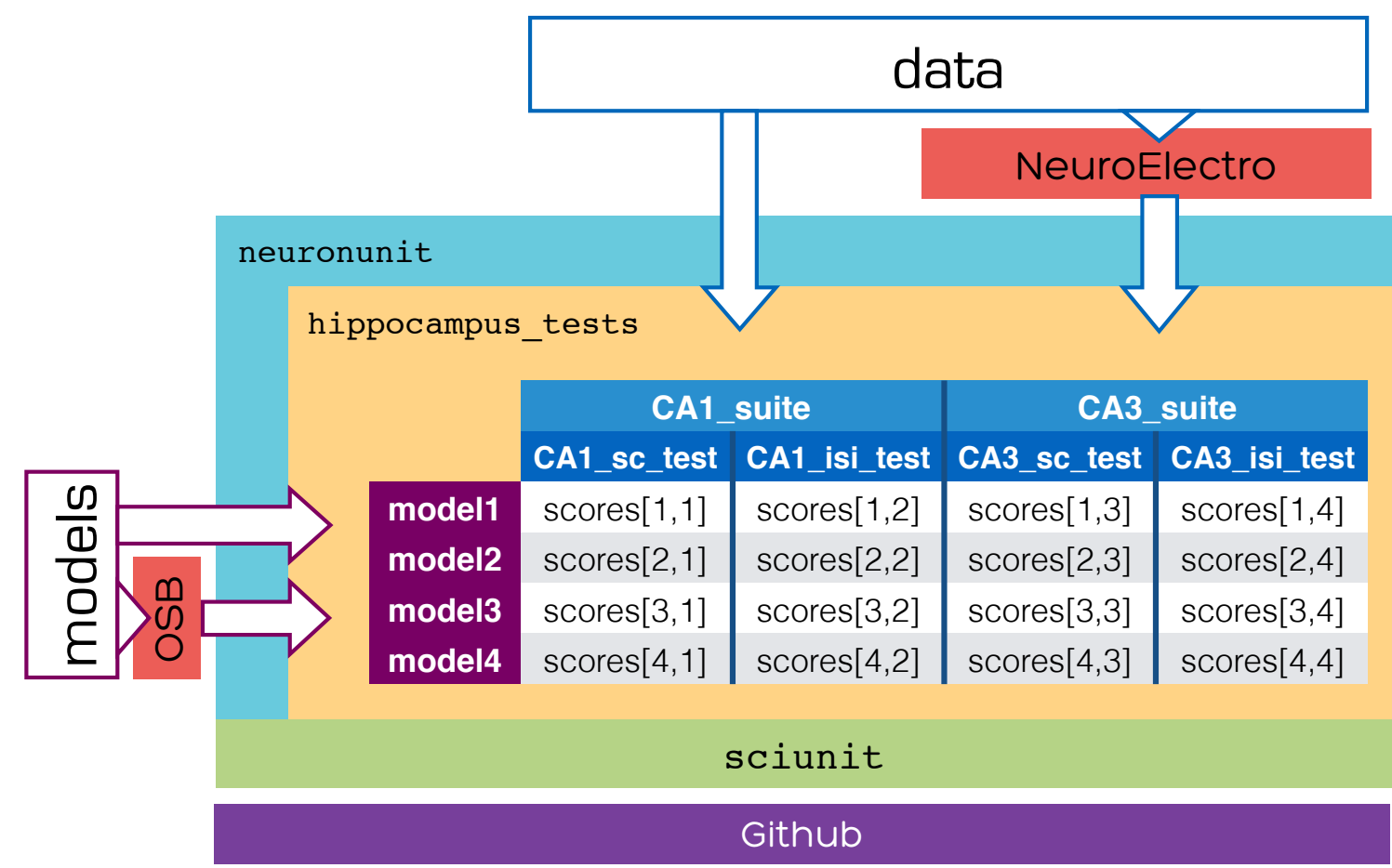

Figure 1. Paper overview. NeuronUnit is set of neurophysiology-specific testing tools built upon the domain-agnostic SciUnit framework. Scientists interested in testing neurophysiological models of particular systems, like the hippocampus, against relevant experimental data can construct test suites in a repository called, for example, hippocampus_tests. Models and data can be added directly or imported, via NeuronUnit, from model repositories like NeuroML-DB or Open Source Brain (OSB) and data repositories like NeuroElectro, The Human Brain Project Microcircuit Portal, or The Allen Institute Cell Types Database. Testing tools and test repositories are developed collaboratively using Github.

trade-off among reduced models of excitable membranes; the "Hopfield" challenge tested techniques for generating neuronal network form given its function ${ }^{9}$; the Neural Prediction Challenge sought the best stimulus reconstructions, given neuronal activity 10 ; the Diadem challenge is advancing the art of neurite reconstruction 11 ; and examples from other areas of biomedical research abound ${ }^{12}$.

\subsection{THE IMPLEMENTATION: SCIUNIT AND NEURONUNIT}

Each of the previous examples leveraged $a d$ hoc infrastructure to support model validation. While the specific criteria used to evaluate models can vary widely between modeling domains, the underlying methodology is common and therefore should be implemented once and re-used. Recognizing this, and inspired by unit testing practices, we previously developed a discipline-agnostic framework for developing model validation test suites called SciUnit ${ }^{3}$ (available from http://sciunit.scidash.org). SciUnit is now used in over a dozen scientific projects ${ }^{5 / 6] 13}-29$ including The Human Brain Project ${ }^{[30 \mid 31}$.

SciUnit contains validation logic common across scientific disciplines. But a particular discipline, such as neurophysiology or neuroanatomy, might have more specialized logic associated with it that can be shared amongst its sub-disciplines (e.g. hippocampal neurophysiology). We have observed a collaborative workflow where this common testing logic has been developed in common repositories on the social coding service GitHub (http://github.com) 6|15|18-24|27-29. For neurophysiological and neuroanatomical tests of single neurons, we have developed such a repository, called NeuronUnit. This repository contains common testing logic as well as bridges to existing neuroinformatics infrastructure to make it easy to import existing datasets and models. In particular, we will describe how models described using 
NeuroML ${ }^{32}$ and provided freely by NeuroML-DB $B^{\frac{\sqrt{33}}{3}}$ or the Open Source Brain Project (OSB $\frac{\sqrt{34}}{4}$ ), can be validated against experimental data in a fully automated fashion, using published data curated by the NeuroElectro Project $\frac{3536}{36}$, the Allen Institute Cell Types database $\frac{37}{37}$, or the Human Brain Project neocortical microcircuit portal 38 .

Investigators in particular sub-disciplines, for example hippocampal neurophysiologists, can use this common logic to select relevant models and tests, forming focused test suites in common repositories 2021 . The end result of this workflow is a table like the one shown in Figure 1, where submitted models can be compared based on the scores they achieve on different selected tests. These tables serve as a summary of the status of a modeling community-just as the results from a suite of traditional unit tests serve as a summary of the status of a software project-and help scientists answer the big questions from section 1.1 more easily, thoroughly, and accurately.

\section{VALIDATION TESTING WITH NEURONUNIT}

\subsection{EXAMPLE: TESTING THE RHEOBASE CURRENT}

We begin by constructing a hypothetical example SciUnit test suite that could be used in neurophysiology. Suppose we have collected data from an experiment where current stimuli (measured in pA) are delivered to neurons of a particular subtype, while the somatic membrane potential of each stimulated cell (in $\mathrm{mV}$ ) is recorded and stored. A model claiming to capture this neuron subtype's membrane potential dynamics must be able to accurately predict a variety of features observed in these data. For neuron physiology, these features have been described and documented at length $36 / 37 / 39 / 40$.

One simple validation test would ask candidate models to predict the rheobase current, i.e. the minimum amplitude square current pulse needed to evoke at least one action potential, as shown in Fig. 2 . After some agreement is reached about protocol design (how long of a square pulse, whether to do a series of fixed current steps or a binary search, what resolution to use, etc.) that protocol can be implemented (lines 15-34), and goodness-of-fit can be measured by calculating a z-score (among other possibilities) given by the difference between the predicted rheobase and the mean observed rheobase (e.g. across cells) divided by the standard deviation of the observed rheobase. The totality of one possible implementation of such a test, using SciUnit is shown in Figure 2. Some of the above considerations-specifically those that are not specific to the problem domain such as calculation of a z-score-are automatically handled by the internal logic of SciUnit through the sciunit. Test class and thus are not shown explicitly. Importantly, in contrast to the simple example in Fig. 2, the actual code for the RheobaseTest in NeuronUnit contains additional logic for speed, handling edges cases and exceptions, using existing standards, and other considerations. However, the code shown in Fig. 2, lines 1-34 is a good representation of the core idea.

\subsection{CONSTRUCTING AND EXECUTING VALIDATION TESTS IN NEURONUNIT}

A SciUnit validation test is an instance (i.e. an object) of a Python class implementing the sciunit. Test interface (Fig. 2, line 1). In order to instantiate it, we must at least provide the observation that parameterizes it (line 40). We emphasize the crucial distinction between the class RheobaseTest, which defines a family of possible validation tests but is not yet tied to any particular experiment observation, and the particular instance my_rheobase_test defined on lines 43-44, an individual validation test parameterized by a particular set of observed experimental data, such as from cells recorded in one investigator's lab.

Alongside this rheobase test, we might also specify a number of other tests capturing different features of the data to produce a more comprehensive suite. For example, the QSNMC defined 17 other validation criteria in addition to one based on the overall spike count, capturing features like spike latencies (SL), mean subthreshold voltage (SV), interspike intervals (ISI) and interspike minima (ISM) that can be 
bioRxiv preprint doi: https://doi.org/10.1101/665331; this version posted June 9, 2019. The copyright holder for this preprint (which was not certified by peer review) is the author/funder, who has granted bioRxiv a license to display the preprint in perpetuity. It is made available under aCC-BY-NC-ND 4.0 International license.

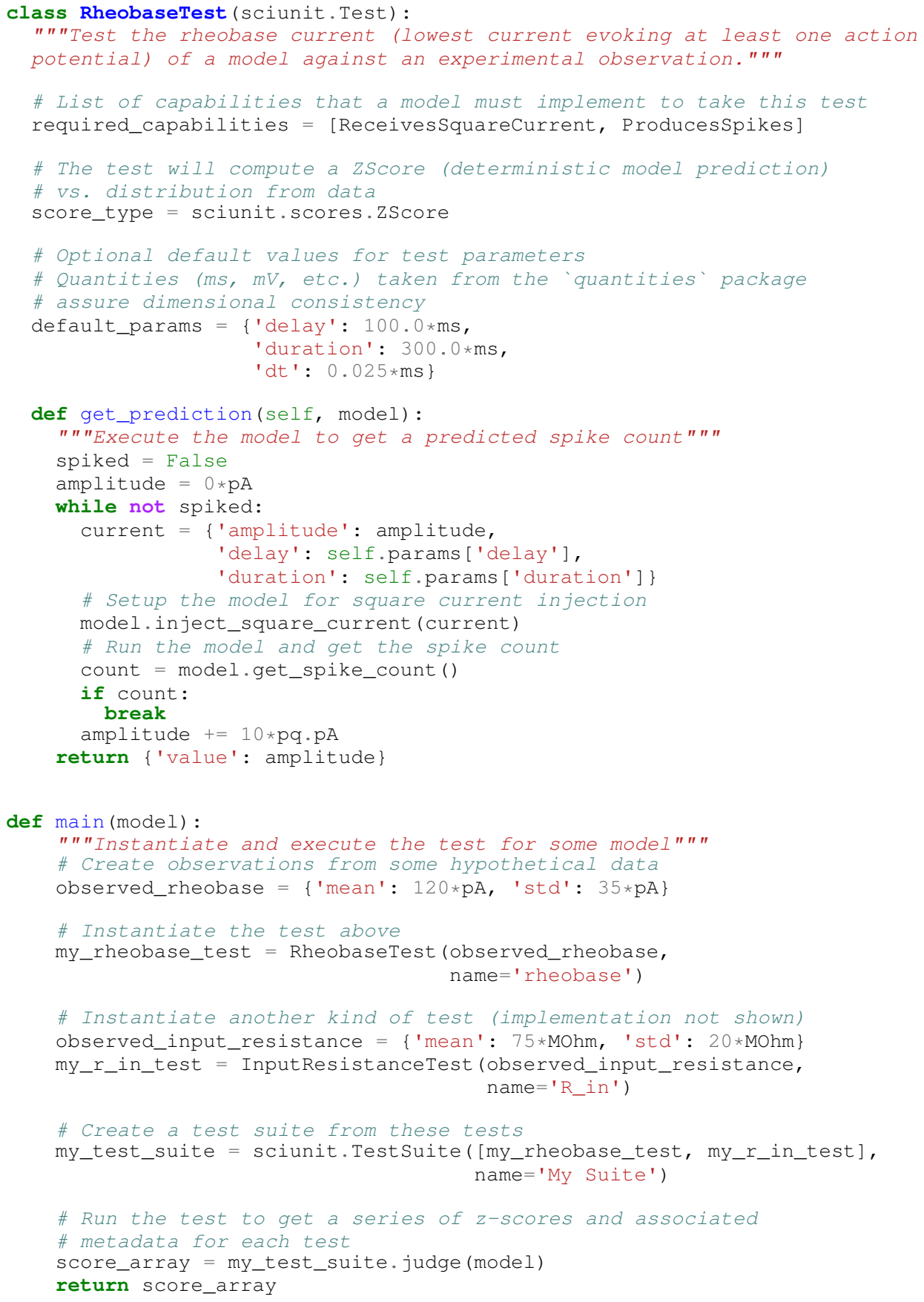

Figure 2. A toy example of a single neuron rheobase test class implemented directly using SciUnit without NeuronUnit. Because this implementation contains logic common to many different systems, NeuronUnit was developed to provide a simpler and more robust means to deliver it.

extracted from the data given appropriate stimulation protocols ${ }^{8}$. On lines $47-48$ we create the observation and test corresponding to one other possible criterion: the input resistance of the cell. The QSNMC authors defined a combined metric favoring models that broadly succeeded at meeting these criteria, to produce an overall ranking. Such combinations of tests, including any weights assigned to each, constitute a test suite, handled by sciunit. Testsuite, as shown in lines 52-53. 
Communities have built SciUnit test repositories capturing the criteria used in their subfields of neuroscience $6|15| 18-24 / 27-29$. . Consequently, test generation for a particular system of interest often requires simply instantiating a previously-developed class with particular experimental parameters and data. We developed NeuronUnit (http://github.com/scidash/neuronunit) to define many such classes of models and tests for neurophysiology and neuroanatomy. In contrast to the toy example in Figure 2 used for illustration, these tests are thoroughly unit-tested (in the traditional software development sense) to handle alternative parameter specifications, edge cases, numerical issues, etc. NeuronUnit currently implements nearly 50 such test classes. Particular instantiated tests, such as a hypothetical CA1_rheobase_test, can then be derived from these classes in suite repositories (e.g. hippocampus_tests, as shown in Figure 1).

\subsection{CAPABILITIES THROUGH ELEPHANT}

All test classes, by virtue of inheriting from sciunit. Test, must contain a get_prediction method (Fig. 2, line 19) that receives a candidate model as input and produces a prediction dictionary as output. Other methods such as compute_score can be provided, or they can default to the methods associated with the parent test class or the provided score type. To specify the interface between the test and the model, the test author provides a list of capabilities in the required_capabilities attribute (Fig. 2. line 6). Capabilities are simply classes describing methods that a test needs a model to implement so that the test can provide inputs and generate relevant predictions using the model. They are analogous to interfaces in e.g. Java. In Python, capabilities are written as classes with unimplemented members. The NeuronUnit capabilities required by the test in Fig. 2 are shown in truncated form in Fig. 3 . The test in Figure 2 uses specific methods of these capability in lines 28 and 30 to produce a spike count prediction for each input current. When a model cannot take a test because of a missing capability, a NotApplicable score is returned (e.g. spiking neuron models describing governed entirely by point processes cannot predict subthreshold voltages) when the test is executed. Thus models which are a poor match to the data can be distinguished from models which cannot even in principle match the data.

Elephant ${ }^{41}$ is a Python library supporting tasks associated with analysis of neural data (or model output), such as membrane potential time series, spike trains, etc. as described by the neuron electrophysiology object library $\mathrm{Neo}^{42}$. It is an open source and actively developed project, containing reliable algorithms on which to base neurophysiology tests. NeuronUnit uses Elephant to give models a path for implementing a variety of capabilities used by NeuronUnit tests. For example, the NeuronUnit capability HasSpikeTrain requires that a method named get_spike_train be implemented, which means that it must (by whatever means) return a Neo SpikeTrain object corresponding to the train of spikes produced by the model under some conditions, e.g. in response to a certain stimulus. NeuronUnit models aiming to implement this capability can do so in part by calling the Elephant method AnalogSignal .threshold_detection, which takes a Neo AnalogSignal object (e.g. a membrane potential time series) and returns a Neo SpikeTrain object (Fig. 4, lines 20-26). This reduces the problem of generating the spike train to one of obtaining a membrane potential time series, which might plausibly vary across models. The membrane potential can be obtained by calling the get_membrane_potential method of the HasMembranePotential capability, with a somewhat more complex and nested implementation that also relies on Neo objects (Fig. 4, lines 8-18). Elephant is used in this manner to implement a variety of NeuronUnit capabilities for NeuronUnit model classes, without requiring that the modeler do so explicitly (though they can override these if they wish in their own model class definitions). This also helps neuroscientists by directing them to a set of common utility functions (in Elephant) and data object types (in Neo) that they can use, knowing that they are well-tested and accepted by the community.

In SciUnit, the judge method (Fig. 2, line 57) checks that these capabilities are implemented by the model, uses them to extract a prediction via get prediction (Fig. 2, lines 19-34), to compute a score indicating model/experiment agreement (not shown) and return a score. In the example from Fig. 2 a zscore was specified (line 10), so an instance of sciunit. scores. ZScore is computed and returned. In 
bioRxiv preprint doi: https://doi.org/10.1101/665331; this version posted June 9,2019 . The copyright holder for this preprint (which was not certified by peer review) is the author/funder, who has granted bioRxiv a license to display the preprint in perpetuity. It is made available under aCC-BY-NC-ND 4.0 International license.

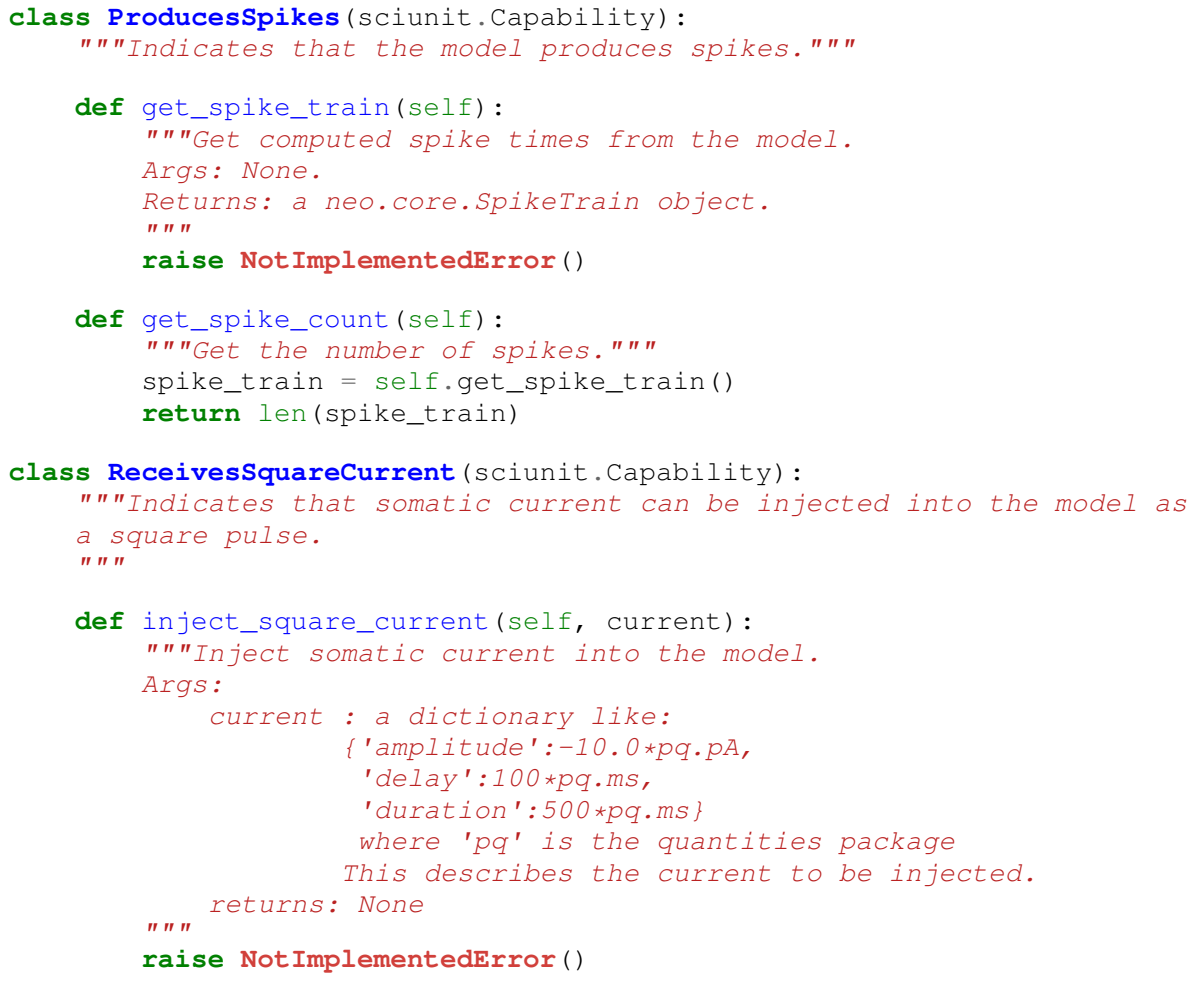

Figure 3. Truncated versions of two capability classes describing the methods (and inputs and outputs) that a model must implement in order to take a test that requires either the ProducesMembranePotential or ReceivesCurrent capability (or both) of the models that it judges. All methods in each capability must have an implementation for the model to satisfy the requirement, but some (see get_spike_count) are automatically implemented by the implementation of others (see get_spike_train). NeuronUnit defines many such capabilities, and separately some reference implementations of those capabilities using various simulator backends including NEURON in conjunction with the Elephant analysis library to, for example, extract a spike train from a membrane potential time series, or a membrane potential time series from a model simulation.

addition to the score itself, the returned score object may also contain metadata, via the related_data parameter, for those who may wish to examine the result in more detail later.

\subsection{MODELS IN NEUROML}

Capabilities are implemented by models. In SciUnit, models are instances of Python classes that inherit from sciunit. Model. Like tests, the class itself represents a family of possible models. A particular model is an instance of such a class, parameterized by the arguments of the constructor

NeuroML is a standardized model description language for neuroscience ${ }^{32}$. It allows many neurophysiological and neuroanatomical models to be described in a simulator-independent fashion. Some simulators, notably the popular NEURON package ${ }^{43}$, can seamlessly export model specifications as NeuroML. Because NeuroML is an XML specification, model descriptions can be verified for correctness and queried for arbitrary information. The latter facilities allow model parameters and capabilities to be automatically determined and also mapped to appropriate variables or functions in simulators. A number of popular simulators support execution of NeuroML models, including NEURON, GENESIS ${ }^{44}$, NEST ${ }^{45}$, and MOOSE 46 . Additionally, over 1500 cell models spanning dozens of cell types can be obtained in NeuroML format from NeuroML-DB (http://neuroml-db.org).

To take advantage of the transparency and machine-readability of models described using NeuroML, NeuronUnit provides a sciunit. Model subclass called NeuromLModel. It can be instantiated directly 
bioRxiv preprint doi: https://doi.org/10.1101/665331; this version posted June 9, 2019. The copyright holder for this preprint (which was not certified by peer review) is the author/funder, who has granted bioRxiv a license to display the preprint in perpetuity. It is made available under aCC-BY-NC-ND 4.0 International license.

\section{Gerkin et al.}

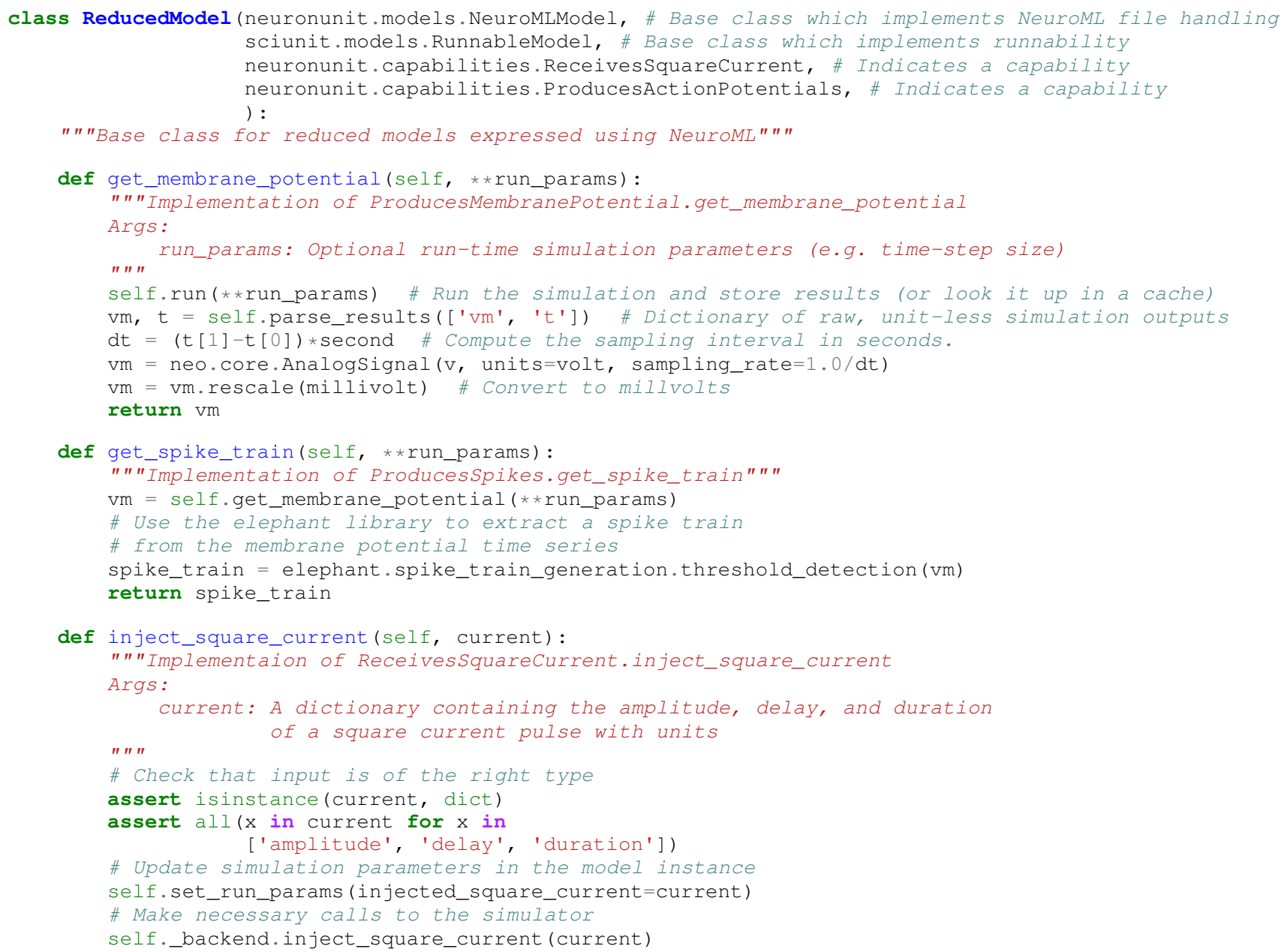

Figure 4. A class for reduced models. This class provides simple implementations of the capabilities required by the NeuronUnit test outlined in Fig. 2 and other tests requiring such capabilities. The constructor, which is inherited from NeuroMLModel and thus not shown, takes a file path or URL to a NeuroML file, and an optional simulator backend.

or sub-classed (useful when additional capabilities need to be manually exposed, for example) to generate a model suitable for testing (Fig. 4). One creates such an instance of a NeuromLModel by passing the path or URL to a NeuroML model file to its constructor.

Figure 4 shows a truncated version of such a model class which can support the execution of models described by NeuroML files (by inheriting from neuronunit. models. NeuroMLModel and which implements the capabilities described in Fig. 3. The implementation of capabilities may rely on third party libraries which have been established for specific tasks in the analysis of neural data (real or simulated). For example, as described above the conversion of a simulated membrane potential time series to a discrete spike train is handled by a function from the Elephant library 41 . Altogether, this model is sufficient to run any test requiring (at most) those capabilities, such as the rheobase test in Fig. 2 or a number of other tests of the features or dynamics of membrane potential time series or spike trains.

\subsection{SIMULATOR BACKENDS AND CACHING}

Simulation itself is handled via one of several simulator Backend classes. These backends implement a bridge between the model class and the simulator. In some cases, the backend may simply use existing 
Python bindings provided by the developers of the simulator. The backend is selected when the model is instantiated (Fig. 4, see caption). In principle, simulating the same conceptual model (e.g. a single NeuroML file) using different simulator backends may produce the same results, and therefore the same test scores, although this cannot be guaranteed. Furthermore, there may be no adequate route for simulating the model described by a NeuroML file on a given simulator, in which case a backend for a more appropriate simulator should be selected.

NeuronUnit currently provides backends for (1) jNeuroML, a NeuroML reference simulator and (2) NEURON, the most widely used simulator for neuron models. Other backends are in development. PyNN is a python modeling library for networks of simple neurons with both broader and deeper coverage of simulators than NeuronUnit offers ${ }^{47}$. Therefore one potential route to broader coverage of simulators in network models would be to create a backend which wraps model interactions in PyNN calls, re-using PyNN's simulator communication infrastructure.

The base Backend class supports caching, such that repeated calls to run the same simulation under the same parameters will simply looks up simulation outputs in the cache rather than unnecessarily run the simulation again. This frequently occurs when different tests require the same simulation outputs in order to compute features. For example, a test of the width of an action potential requires the same simulation output as the test of the height of an action potential. Therefore only the first of these tests causes the simulation to be run. The second of these tests will operate on cached simulation output to compute a different feature.

\subsection{TEST SUITES AND SCORE MATRICES}

A collection of tests intended to be run on the same model can be put together to form a test suite, as in Fig. 2, lines 51-53. Like a single test, a test suite is capable of judging one or more models. To make the idea of a test suite more concrete, we created a test suite from six NeuronUnit tests that compare basic electrophysiological properties of model neurons to their experimental counterparts. Specifically, we instantiated these tests using data for olfactory bulb mitral cells taken from http://neuroelectro.org $35 \mid 36$. We then executed this test suite against seven published models of olfactory bulb mitral cells. The output of this test execution is a ScoreMatrix containing a score for each model on each test (Fig. 5). A ScoreMatrix is a subclass of a Pandas dataframe ${ }^{48}$ through which scores for a given model or a test can be summarized, visualized, or probed for further details. Each row or column of a ScoreMatrix is a ScoreArray (a subclass of a Pandas series) summarizing the scores for one model or test, as returned in line 57 of Fig. 2.

\subsection{TRAINING AND OPTIMIZATION}

Some models could in principle match experimental observations, but not under the current set of parameters. Optimizing a model's parameters through testing could produce a model which is a better description of the underlying system. We have been developing test-driven model optimization in NeuronUnit using a combination of Scoop ${ }^{49}$ (for parallelism), DEAP ${ }^{50}$ (for genetic optimization), and BluePyOpt ${ }^{51}$. This allows models to be optimized against suites of NeuronUnit tests, either weighting the tests to produce "best" models for the suite, or retaining the entire non-dominated set, i.e. all model parameterizations that are not strictly worse than some other parameterization on every test. This latter option may be important to avoid producing models which have the greatest correspondence to the mean value of all experimental data features for a neuron type, but which do not correspond to the behavior of any particular neuron ${ }^{52}$. We have used this feature internally to optimize reduced neuron models against suites of tests of electrophysiological properties in order to produce sets of reduced models for each of several major cell types in the brain. We plan to release these optimized models as"plug and play" options for investigators who wish to build circuit models containing several distinct, realistic, neuron subtypes. However, this features is not yet production ready. 


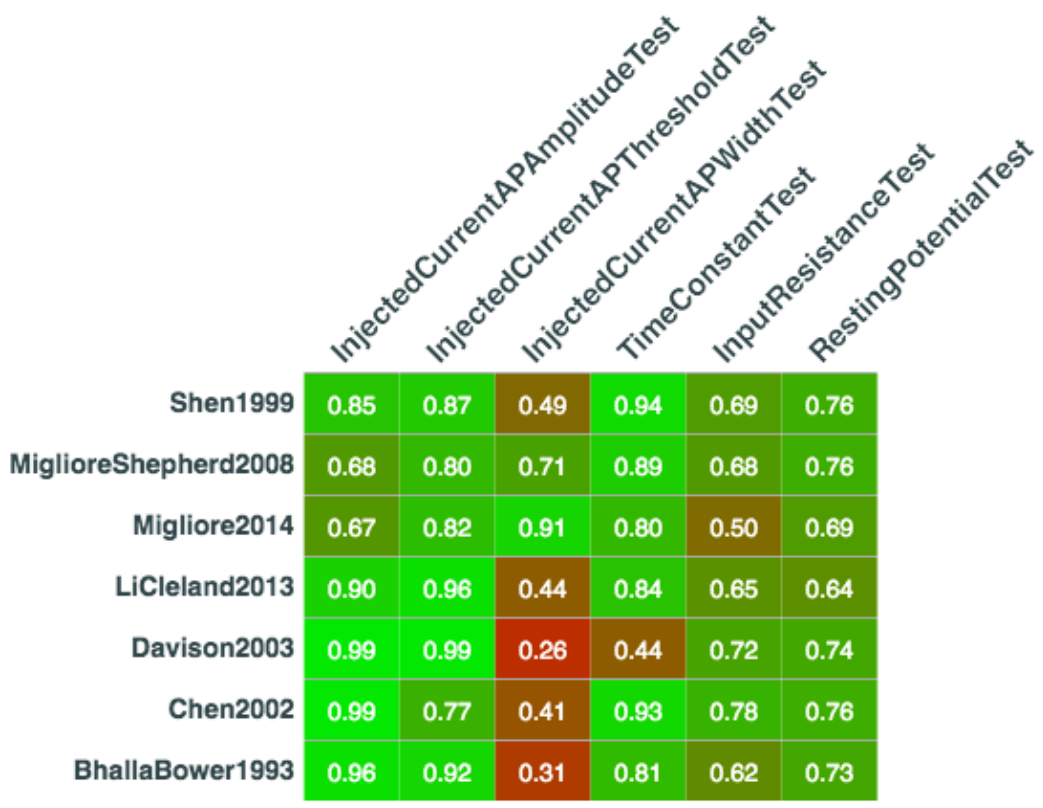

Figure 5. A score matrix for a SciUnit test suite, visualized as a hyperlinked table inside a Jupyter notebook stored in a test repository at http://github.com/ justasb/mitralsuite Individual elements of this score matrix can be further probed to see test and model details as well as simulation outputs or experimental data that gave rise to these scores. For ease of visualization, each raw score (a z-score comparing model output to the experimental data distribution) has been automatically converted into a normalized score ranging from 0 (lowest possible agreement) to 1 (best possible agreement, i.e. that feature of the model's output is equal to the mean of the same feature in the experimental data distribution).

\subsection{MODELS IN OPEN SOURCE BRAIN}

Open Source Brain $\left(O S B^{34}\right)$ curates many models described in NeuroML, and is a hub for collaborative modeling. In constrast to NeuroML-DB, OSB is intended to be a platform for on-going model development. OSB-curated projects have been converted from their native format (e.g. NEURON) into NeuroML. To ensure that the NeuroML model is faithful to the original simulation, OSB verifies concordance between model output (beginning with the NeuroML description) and reference output (from native simulator source files or published figures) for each model. Thus, OSB is an excellent source of modeling projects that, in addition to being open source, are known to be accurately described. This also highlights the distinction between verification activities, performed by OSB to ensure that a model description operates correctly, and validation activities, performed using NeuronUnit to check the model's predictions against data. Both are important activities. NeuronUnit also implements an OSBModel class for those who wish to start testing an active modeling project on OSB.

\subsection{MORPHOLOGY TESTS}

Some neuron models aim to have realistic neuron morphologies. NeuronUnit provides a set of morphology tests that use pyLMeasure ${ }^{53}$, a python interface to the LMeasure ${ }^{54}$ tool for generating morphological features from both model neurons and reconstructions of real neurons. These tests can be parameterized with either features of some real neuron's morphology, or distributions of such features across ensembles of real neurons. In order to take these tests, models must implement a Produces SWC capability, which means the model class must have a means of generating a standard .swc file (as used in neurite reconstructions) corresponding to its morphology. For models expressed in NeuroML or NEURON, this is an easy capability to implement. 
bioRxiv preprint doi: https://doi.org/10.1101/665331; this version posted June 9,2019 . The copyright holder for this preprint (which was

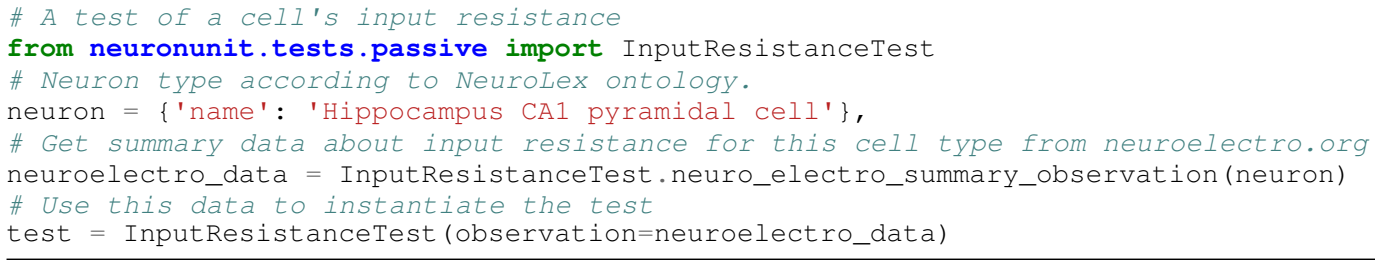

Figure 6. Tests can be built directly from cell-type-specific data automatically pulled from http://neuroelectro.org

\section{DATA SOURCES}

In the examples in Fig. 2, tests were provided data explicitly (e.g. lines 40 and 47). However, experimental data about neurons are also available from existing community resources in standardized formats. In this section, we will show how to use this existing infrastructure to parameterize tests with published data automatically.

\subsection{NEUROELECTRO}

The NeuroElectro project (http://neuroelectro.org) is an effort to make all published data on single cell neurophysiology available in a machine-readable format ${ }^{55}$. Currently, up to 47 electrophysiological properties are available for 235 cell types, gathered from over 2000 single pieces of published data extracted from tables in published papers. NeuroElectro uses the NeuroLex.org ontology $\sqrt{56}$ to identify specific neuron types and specific electrophysiological properties, and indexes corresponding data values from the literature. We have made it easy to parameterize the test families in NeuronUnit using data extracted from the NeuroElectro API. Tests can be based upon data from single journal articles, or from ensembles of articles with a common theme (e.g. about a particular neuron type). The latter is illustrated in Figure 6. Once data has been retrieved from NeuroElectro, associated statistics (e.g. mean, standard error and sample size) are extracted to parameterize tests (e.g. a test of a model cell's input resistance). Data from NeuroElectro can be used to validate a variety of basic electrophysiological features of neuron models, including spike width, resting membrane potential, after-hyperpolarization amplitude, etc. As NeuroElectro is the only public, curated source of such data for many neuron types, it represents a key resource facilitating test construction. However, in selecting data from NeuroElectro (either averaged data across all reports, or data from selected reports) for test construction, one is implicitly assuming that it represents the kind data that ought to be binding in model development. For some models, data obtained in this way ought not to guide model development, and other data sources will need to be identified and used for test construction.

\subsection{OTHER PUBLIC DATA SOURCES}

For those interested primarily in neurons from either visual cortex or somatosensory cortex, NeuronUnit can also automatically build tests using data from The Allen Institute's Cell Types Database ${ }^{37}$ and The Human Brain Project's Neocortical Microcircuit Collaboration Portal ${ }^{38}$, respectively. Neuronunit's bbp and aibs modules provide the means to communicate with the corresponding APIs or download data directly, to extract recordings that can be used to compute the features used in observations that will parameterize a NeuronUnit test. 


\section{DISCUSSION}

Neuroscientists can use NeuronUnit to easily create test suites tailored to specific neuronal systems of interest. For example, investigators interested specifically in testing models of cells in the hippocampus would use NeuronUnit to create tests in a GitHub repository called e.g. hippocampus_tests, containing scripts or Jupyter notebooks containing the steps needed to instantiate and execute validation tests parameterized by empirical data from the hippocampus against corresponding hippocampal cell models. Many of the other features and details of NeuronUnit, including specific examples for developers, can be found at http://github.com/scidash/neuronunit.

\subsection{DIFFERENCES OF OPINION}

Validation criteria are subject to debate (indeed, the QSNMC criteria changed between 2007 and 2008 due to such debates), and identification and correction of flawed methodology is becoming increasingly common $57-59$. Statisticians and other scientists interested in improving how goodness-of-fit is measured can simply fork the NeuronUnit repository, modify the logic in the relevant test families and submit a pull request for evaluation by the community. Rather than attempting to persuade a large number of scientists that the methods used in canonical papers need to be changed, statisticians need only propagate a change to the testing logic used by the community. Once the testing logic has been changed, all models can be immediately evaluated against the new metrics. Those who wish to identify statistical best-practices for measuring goodness-of-fit need only refer to the tests in these repositories.

\subsection{COMMUNITY CONTRIBUTIONS}

To submit a new model for testing, one can similarly fork a test repository (e.g. hippocampus_tests), add a new model and test it locally using the tests in the repository. When satisfied with parameter choices, one can submit a pull request to the upstream repository. Once a model is in the repository, it can be evaluated on an ongoing basis against the latest accepted collections of tests, parameterized by the latest data, using the latest statistical best practices, without the participation of the original modeler, assuming only that the capabilities required by the tests stay stable.

GitHub is an excellent platform for this kind of activity, and is becoming a widely-used tool for scientific collaboration, reproducibility, and transparency 60 . We anticipate that other communities within neuroscience (and indeed, within science more broadly) will develop repositories similar to NeuronUnit that provide a common, collaboratively-developed vocabulary of test families and capabilities relevant to their domain, and bridge relevant standardization efforts and infrastructure. Using these common repositories, smaller groups of investigators interested in particular systems of interest will develop tests and models in test repositories like hippocampus_tests. Each research community can develop suitable quality standards and processes for merging pull requests.

\subsection{PUBLIC ASSESSMENT}

The most visible result of these efforts will be tables that allow investigators to represent, formally, the state of model development in their field, as judged against corresponding experimental data. The ScoreMatrix is an implementation of such a table, and when rendered in a Jupyter notebook it appears similar to the one shown in Figure 5. Thus a Jupyter notebook, in addition to representing a reproducible workflow for testing, can also be used to visualize the test outputs themselves.

We are also developing a lightweight portal, SciDash, that directs investigators to the canonical tests in their field in order to prevent fragmentation and encourage community participation. SciDash will also facilitate viewing test results without needing to clone test repositories and run tests locally for exploratory purposes. Finally, SciDash will support the scheduling and execution of tests for those who prefer a web 
interface to a notebook or IDE. SciDash will not be a place for the development of models, as existing services such as OSB and NetPyne ${ }^{61}$ are already well-suited to that purpose.

\subsection{MANUALLY CREATING NEW MODELS, CAPABILITIES, AND TESTS}

NeuronUnit provides base classes to enable rapid generation of models, capabilities, and tests for neurophysiology and neuron morphology data. However these objects can also be created from scratch, requiring only adherence to the SciUnit interface as exemplified in sec. 2. For example, a Model could implement an integrate capability method by wrapping execution of a MATLAB script and a get_spikes capability method by parsing a resulting .csv file on disk; a Test could be initialized using empirical spike rates collected in the lab. While this does not meet our idealized vision of collaborative development and testing, in practice this may be a common scenario.

\subsection{PARTICIPATION FROM MODELING COMMUNITIES}

Modelers may not want to formally expose their models to tests via capabilities, a requirement for testtaking. We anticipate four solutions: First, we will emphasize to the community that wrapping existing model code that satisfy a capability is quite simple, requiring as little as one line of code. Importantly, the modeler is not required to expose or rewrite any internal model flow control in almost all cases. Second, we support multiple simulation environments automatically by using NeuroML ${ }^{32}$, and other simulatorindependent model descriptions are possible for other domains. Automated generation of NeuroML from native model source code is in development (Gleeson, personal communication); for the popular NEURON simulator ${ }^{43}$, this functionality is already implemented and in use for some model components. This minimizes modeler effort for a large (at least 1500) and growing number of neuronal models that are not already in NeuroML-DB. However, models converted from native simulator code to NeuroML must still be examined manually to ensure that nothing has been lost in translation. Third, modelers have an incentive to demonstrate publicly their models' validity. Participation in public modeling competitions demonstrates that this incentive is sufficient to recruit several participants. We plan to assist with such competitions by advocating for use of SciUnit as the underlying technical substrate in the future. Currently, we are in discussion with field specialists to organize competitions for spiking neuron models (a sequel to the QSNMC), spike-sorting algorithms, calcium imaging spike time reconstruction, braincomputer interfaces, neural network function prediction, and seizure prediction. Fourth, modelers have an incentive to use SciUnit or NeuronUnit during development (see TDD, Sec. 1) to ensure that ongoing development preserves correspondence between model and data. A popular test suite can represent a "gold standard" by which progress during development is judged, even within a single project.

\subsection{PARTICIPATION FROM EXPERIMENTAL COMMUNITIES}

Experimentalists may not want to write tests derived from their data. We anticipate four solutions: First, we will emphasize that tests do not require releasing entire datasets; a test consists only of a list of required capabilities (for selecting eligible models), and sufficient statistics suitable for executing the scoring logic. This can simply be means and standard deviations in many cases, such as the examples in this paper. Most scoring logic will consist of a few calls to capability methods followed by a standard statistical test, which can be implemented once in SciUnit or a discipline-specific repository like NeuronUnit. Second, data-sharing is becoming accepted. If data is available from a community repository, as many granting agencies are increasingly requiring, then tests can often be generated automatically using bridges developed collaboratively by the community. Third, an incentive to write tests for one's data exists: the ability to identify models that give the data clear context and impact. If a new piece of data invalidates a widelyaccepted model, this can be cited in publications. Fourth, one can compare new data against existing data by simply creating a data-derived model that directly generates "predictions" by draws from the existing 
dataset directly. Thus the model validation procedures described in this paper can also be used to perform data validation - that is, check the goodness-of-fit of new data with old data.

\subsection{OCCAM'S RAZOR}

All things being equal, simpler models are better. Model complexity, though fundamentally a subjective measure, has many correlates $\frac{62}{2}$, including: 1) model length; 2) memory use; 3) CPU load; 4) \# of capabilities. Python packages for reporting these complexity measures exist ${ }^{63}$, and can be used in conjunction with test scores themselves to visualize the model validity vs complexity tradeoff, for example as a scatter plot, with the "best" models being in the high validity / low complexity corner of the plot. The set of models which dominate all others, i.e. that have the highest validity for a given complexity, can be represented as a "frontier" in such a scatter plot (e.g. as used in the symbolic regression package Eureqa ${ }^{64}$ ).

\subsection{EXPANSION INTO OTHER AREAS OF NEUROSCIENCE}

NeuronUnit largely covers the neurophysiology and morphology of single neurons. We would like to extend both it and the larger SciUnit effort across neuroscience and the other biological sciences. The SciUnit framework is discipline-agnostic, so community participation and model description are the only obstacles. Community participation begins with enumerating the capabilities relevant to a sub-discipline, and then writing tests. Model description can expand within NeuroML (which already covers multiple levels within neuroscience) and tools for capability implementation can incorporate libraries for neuroimaging (NiBabel, http://nipy.org/nibabel), neuroanatomy (NeuroHDF, http://neurohdf.readthedocs.org) and other sub-disciplines. SBML will enable expansion beyond neuroscience, facilitated by parallel efforts among NeuroML developers to interface with it (Crook, unpublished). SciUnit has already been used extensively at several levels from ion channels to behavior in the OpenWorm project ${ }^{56 / 17}$, which through open, collaborative development seeks to model the entire organism $C$. elegans.

\subsection{PROJECT DEVELOPMENT}

Gewaltig and Cannon describe the maturity of a computational software project ${ }^{65}$ as "review ready", "research ready", or "user ready". The development of the underlying SciUnit framework is complete ${ }^{3}$, and it provides the full interface necessary for continued development of domain-specific unit-testing frameworks - it is research ready if not user ready. NeuronUnit itself is "review ready" - we invite others to participate in building it out to satisfy the use cases they encounter. Meanwhile, corresponding test suite repositories on GitHub, containing NeuronUnit-based tests, do not even reach this stage (with few exceptions ${ }^{21}$ ). However, many neuroscience projects are realizing this vision using SciUnit directly $5 / 6[15-2024-29$. We plan to continue creating such repositories; more importantly we invite interested parties to create their own, either to guide in-house model development or to assess larger pools of established models.

\section{DISCLOSURE/CONFLICT-OF-INTEREST STATEMENT}

The authors declare that the research was conducted in the absence of any commercial or financial relationships that could be construed as a potential conflict of interest. 


\section{ACKNOWLEDGEMENTS}

We thank Jonathan Aldrich, Shreejoy Tripathy, and Padraig Gleeson, and MetaCell LLC for their support of this project. The work was supported in part by grant R01MH081905 and R01MH106674 from the National Institute of Mental Health, and grant R01MH106674 from the National Institute for Biomedical Imaging and Bioengineering. The content is solely the responsibility of the authors and does not necessarily represent the official views of the National Institutes of Health.

\section{REFERENCES}

[1]David Donoho, Arian Maleki, Inam Rahman, Morteza Shahram, and Victoria Stodden. 15 years of reproducible research in computational harmonic analysis. IEEE Computing in Science and Engineering, 11:818, 2008.

[2]Arif E. Jinha. Article 50 million: an estimate of the number of scholarly articles in existence. Learned Publishing, 23(3):258-263, July 2010.

[3]Cyrus Omar, Jonathan Aldrich, and Richard C. Gerkin. Collaborative infrastructure for test-driven scientic model validation. ICSE: New Ideas and Emerging Results, Accepted:1-4, 2014.

[4]Kent Beck. Test Driven Development: By Example. Addison Wesley, 2003.

[5]Gopal P Sarma, Travis W Jacobs, Mark D Watts, S Vahid Ghayoomie, Stephen D Larson, and Richard C Gerkin. Unit testing, model validation, and biological simulation. F1000Res., 5:1946, 2016.

[6]Richard C Gerkin, Russell J Jarvis, and Sharon M Crook. Towards systematic, data-driven validation of a collaborative, multi-scale model of caenorhabditis elegans. Philos. Trans. R. Soc. Lond. B Biol. Sci., 373(1758), September 2018.

[7]Andreas Keller, Richard C Gerkin, Yuanfang Guan, Amit Dhurandhar, Gabor Turu, Bence Szalai, Joel D Mainland, Yusuke Ihara, Chung Wen Yu, Russ Wolfinger, Celine Vens, Leander Schietgat, Kurt De Grave, Raquel Norel, DREAM Olfaction Prediction Consortium, Gustavo Stolovitzky, Guillermo A Cecchi, Leslie B Vosshall, and Pablo Meyer. Predicting human olfactory perception from chemical features of odor molecules. Science, 355(6327):820-826, 2017.

[8]Renaud Jolivet, Felix Schrmann, Thomas Berger, Richard Naud, Wulfram Gerstner, and Arnd Roth. The quantitative single-neuron modeling competition. Biological Cybernetics, 99(4):417-426, November 2008.

[9]J J Hopfield and C D Brody. What is a moment? "Cortical" sensory integration over a brief interval. Proceedings of the National Academy of Sciences of the United States of America, 97(25):1391913924, December 2000. PMID: 11095747.

[10]http://neuralprediction.berkeley.edu/.

[11]http://www.diademchallenge.org.

[12] http://www.the-dream-project.org/.

[13]Russel J Jarvis, Sharon M Crook, and Richard C Gerkin. Parallel Model Optimization against Experimental Neuron Physiology Data with DEAP and NeuronUnit, 2017.

[14]Russel J Jarvis, Sharon M Crook, and Richard C Gerkin. Optimization of Reduced Models against Diverse Experimental Neuron Physiology Datasets with NeuronUnit", CRCNS PI Meeting, Brown University, 2017, 2017.

[15]Robin Gutzen, Michael von Papen, Guido Trensch, Pietro Quaglio, Sonja Grün, and Michael Denker. Reproducible neural network simulations: Statistical methods for model validation on the level of network activity data. Front. Neuroinform., 12:90, December 2018.

[16]SciDash. http://dash.scidash.org. Accessed: 2019-2-26.

[17]Gopal P Sarma, Chee Wai Lee, Tom Portegys, Vahid Ghayoomie, Travis Jacobs, Bradly Alicea, Matteo Cantarelli, Michael Currie, Richard C Gerkin, Shane Gingell, Padraig Gleeson, Richard Gordon, Ramin M Hasani, Giovanni Idili, Sergey Khayrulin, David Lung, Andrey Palyanov, Mark Watts, and Stephen D Larson. OpenWorm: overview and recent advances in integrative biological simulation of caenorhabditis elegans. Philos. Trans. R. Soc. Lond. B Biol. Sci., 373(1758), September 2018. 
[18]SubCellUnit: A SciUnit library for data-driven testing of subcellular features.

[19]Basalunit: A SciUnit library for data-driven testing of basal ganglia models.

[20]Andrew Davison. Hippounit: A SciUnit library for data-driven validation testing of models of hippocampus.

[21]Justas Birgiolas. MitralSuite: Validation tests for mitral cell models.

[22]NeuronUnit website. http://neuronunit.scidash.org. Accessed: 2018-7-23.

[23]NeuronUnit (human brain project fork).

[24]Christoph Metzner. ASSRUnit: Validation tests for auditory research.

[25]Christoph Metzner, Tuomo Mäki-Marttunen, Bartosz Zurowski, and Volker Steuber. Modules for automated validation and comparison of models of neurophysiological and neurocognitive biomarkers of psychiatric disorders: ASSRUnit-A case study. Computational Psychiatry, 2:74-91, 2018.

[26]NeuroElectroSciUnit: An open source brain project highlighting some of the potential interactions between OSB and NeuroElectro.org and SciUnit.

[27]Cerebellum-unit.

[28]eFELunit: A SciUnit library for data-driven testing of eFEL features extracted from computational models.

[29]MorphoUnit: A SciUnit library for data-driven testing of neuronal morphologies.

[30]Human brain project home. https://www.humanbrainproject.eu/en/. Accessed: 2018-7-22.

[31]Announcing the winners of the HBP infrastructure voucher call 2018 - news. https://www.humanbrainproject.eu/en/follow-hbp/news/ announcing-the-winners-of-the-hbp-infrastructure-voucher-call-2018/. Accessed: 2019-6-3.

[32]Padraig Gleeson, Sharon Crook, Robert C Cannon, Michael L Hines, Guy O Billings, Matteo Farinella, Thomas M Morse, Andrew P Davison, Subhasis Ray, Upinder S Bhalla, Simon R Barnes, Yoana D Dimitrova, and R Angus Silver. NeuroML: a language for describing data driven models of neurons and networks with a high degree of biological detail. PLoS computational biology, 6(6): e1000815, June 2010. PMID: 20585541.

[33]J. Birgiolas, R.C. Gerkin, S.M. Crook. Rapid selection of NeuroML models via NeuroML-DB.org. Organization for Computational Neuroscence Meeting, 2018.

[34]Padraig Gleeson, Eugenio Piasini, Sharon Crook, Robert Cannon, Volker Steuber, Dieter Jaeger, Sergio Solinas, Egidio D'Angelo, and R Angus Silver. The open source brain initiative: enabling collaborative modelling in computational neuroscience. BMC Neuroscience, 13(Suppl 1):O7, July 2012. PMID: null PMCID: PMC3403499.

[35]Shreejoy J Tripathy, Judith Savitskaya, Shawn D Burton, Nathaniel N Urban, and Richard C Gerkin. NeuroElectro: a window to the world's neuron electrophysiology data. Front. Neuroinform., 8:40, 2014.

[36]Shreejoy J Tripathy, Shawn D Burton, Matthew Geramita, Richard C Gerkin, and Nathaniel N Urban. Brain-wide analysis of electrophysiological diversity yields novel categorization of mammalian neuron types. J. Neurophysiol., 113(10):3474-3489, June 2015.

[37]Overview :: Allen brain atlas: Cell types. https://celltypes.brain-map.org/. Accessed: 2019-6-6.

[38]Srikanth Ramaswamy, Jean-Denis Courcol, Marwan Abdellah, Stanislaw R Adaszewski, Nicolas Antille, Selim Arsever, Guy Atenekeng, Ahmet Bilgili, Yury Brukau, Athanassia Chalimourda, Giuseppe Chindemi, Fabien Delalondre, Raphael Dumusc, Stefan Eilemann, Michael Emiel Gevaert, Padraig Gleeson, Joe W Graham, Juan B Hernando, Lida Kanari, Yury Katkov, Daniel Keller, James G King, Rajnish Ranjan, Michael W Reimann, Christian Rössert, Ying Shi, Julian C Shillcock, Martin Telefont, Werner Van Geit, Jafet Villafranca Diaz, Richard Walker, Yun Wang, Stefano M Zaninetta, Javier DeFelipe, Sean L Hill, Jeffrey Muller, Idan Segev, Felix Schürmann, Eilif B Muller, and Henry Markram. The neocortical microcircuit collaboration portal: a resource for rat somatosensory cortex. Front. Neural Circuits, 9:44, October 2015.

[39]Petilla Interneuron Nomenclature Group, Giorgio A Ascoli, Lidia Alonso-Nanclares, Stewart A Anderson, German Barrionuevo, Ruth Benavides-Piccione, Andreas Burkhalter, György Buzsáki, Bruno Cauli, Javier Defelipe, Alfonso Fairén, Dirk Feldmeyer, Gord Fishell, Yves Fregnac, Tamas F Freund, Daniel Gardner, Esther P Gardner, Jesse H Goldberg, Moritz Helmstaedter, Shaul Hestrin, Fuyuki 
Karube, Zoltán F Kisvárday, Bertrand Lambolez, David A Lewis, Oscar Marin, Henry Markram, Alberto Muñoz, Adam Packer, Carl C H Petersen, Kathleen S Rockland, Jean Rossier, Bernardo Rudy, Peter Somogyi, Jochen F Staiger, Gabor Tamas, Alex M Thomson, Maria Toledo-Rodriguez, Yun Wang, David C West, and Rafael Yuste. Petilla terminology: nomenclature of features of GABAergic interneurons of the cerebral cortex. Nat. Rev. Neurosci., 9(7):557-568, July 2008.

[40]Shaul Druckmann, Sean Hill, Felix Schürmann, Henry Markram, and Idan Segev. A hierarchical structure of cortical interneuron electrical diversity revealed by automated statistical analysis. Cereb. Cortex, 23(12):2994-3006, December 2013.

[41]Overview - elephant 0.6.0 documentation. https://elephant.readthedocs.io/en/latest/overview.html. Accessed: 2019-2-26.

[42]Samuel Garcia, Domenico Guarino, Florent Jaillet, Todd Jennings, Robert Pröpper, Philipp L Rautenberg, Chris C Rodgers, Andrey Sobolev, Thomas Wachtler, Pierre Yger, and Andrew P Davison. Neo: an object model for handling electrophysiology data in multiple formats. Front. Neuroinform., 8:10, February 2014

[43]NT Carnevale and ML Hines. The NEURON Book. Cambridge University Press, Cambridge, UK, 2006.

[44]J.M. Bower and D. Beeman. Constructing realistic neural simulations with GENESIS. Methods Mol. Biol., 401:103-125, 2007.

[45]Marc-Oliver Gewaltig and Markus Diesmann. Nest (neural simulation tool). Scholarpedia, 2(4):1430, 2007.

[46]Subhasis Ray, Raamesh Deshpande, Niraj Dudani, and Upinder S Bhalla. A general biological simulator: the multiscale object oriented simulation environment, moose. BMC Neuroscience, 9(Suppl. 1):93, 2008.

[47]Andrew P Davison, Daniel Brüderle, Jochen Eppler, Jens Kremkow, Eilif Muller, Dejan Pecevski, Laurent Perrinet, and Pierre Yger. PyNN: A common interface for neuronal network simulators. Front. Neuroinform., 2:11, 2008.

[48]Python data analysis library — pandas: Python data analysis library. https://pandas.pydata.org/ Accessed: 2019-6-6.

[49]Philosophy - SCOOP 0.7.2 dev documentation. https://scoop.readthedocs.io/en/0.7/. Accessed: 2019-6-6.

[50]DEAP documentation - DEAP 1.2.2 documentation. https://deap.readthedocs.io/en/master/ Accessed: 2019-6-6.

[51]Werner Van Geit, Michael Gevaert, Giuseppe Chindemi, Christian Rössert, Jean-Denis Courcol, Eilif B Muller, Felix Schürmann, Idan Segev, and Henry Markram. BluePyOpt: Leveraging open source software and cloud infrastructure to optimise model parameters in neuroscience. Front. Neuroinform., 10:17, June 2016.

[52]Astrid A Prinz, Dirk Bucher, and Eve Marder. Similar network activity from disparate circuit parameters. Nat. Neurosci., 7(12):1345-1352, December 2004.

[53]Justas Birgiolas. pylmeasure.

[54]Ruggero Scorcioni, Sridevi Polavaram, and Giorgio A Ascoli. L-Measure: a web-accessible tool for the analysis, comparison and search of digital reconstructions of neuronal morphologies. Nat. Protoc., 3(5):866-876, 2008.

[55]SJ Tripathy, J Saviskaya, RC Gerkin, and NN Urban. NeuroElectro: a database describing the electrophysiology properties of different neuron types. Neuroinformatics Meeting, 2012.

[56]Stephen D. Larson and Maryann E. Martone. NeuroLex.org: an online framework for neuroscience knowledge. Frontiers in Neuroinformatics, 7:18, 2013.

[57]Katherine S Button, John P A Ioannidis, Claire Mokrysz, Brian A Nosek, Jonathan Flint, Emma S J Robinson, and Marcus R Munaf. Power failure: why small sample size undermines the reliability of neuroscience. Nature reviews. Neuroscience, 14(5):365-376, May 2013. PMID: 23571845.

[58]Nikolaus Kriegeskorte, W Kyle Simmons, Patrick S F Bellgowan, and Chris I Baker. Circular analysis in systems neuroscience: the dangers of double dipping. Nature neuroscience, 12(5):535-540, May 2009. PMID: 19396166. 
bioRxiv preprint doi: https://doi org/10.1101/665331; this version posted June 9,2019 . The copyright holder for this preprint (which was not certified by peer review) is the author/funder, who has granted bioRxiv a license to display the preprint in perpetuity. It is made available under aCC-BY-NC-ND 4.0 International license.

[59]Sally Galbraith, James A. Daniel, and Bryce Vissel. A study of clustered data and approaches to its analysis. The Journal of Neuroscience, 30(32):10601-10608, August 2010. PMID: 20702692.

[60]Karthik Ram. Git can facilitate greater reproducibility and increased transparency in science. Source code for biology and medicine, 8(1):7, 2013. PMID: 23448176.

[61]Salvador Dura-Bernal, Benjamin A Suter, Padraig Gleeson, Matteo Cantarelli, Adrian Quintana, Facundo Rodriguez, David J Kedziora, George L Chadderdon, Cliff C Kerr, Samuel A Neymotin, Robert A McDougal, Michael Hines, Gordon Mg Shepherd, and William W Lytton. NetPyNE, a tool for data-driven multiscale modeling of brain circuits. Elife, 8, April 2019.

[62]TJ McCabe. A complexity measure. IEEE Transactions on Software Engineering, 2(4):308-320, 1976.

[63]Welcome to radon's documentation! — radon 2.4.0 documentation. https://radon.readthedocs.io/en/ latest/. Accessed: 2019-6-6.

[64]Michael Schmidt and Hod Lipson. Distilling free-form natural laws from experimental data. Science (New York, N.Y.), 324(5923):81-85, April 2009. PMID: 19342586.

[65]Marc-Oliver Gewaltig and Robert Cannon. Current practice in software development for computational neuroscience and how to improve it. PLoS Comput Biol, 10(1):e1003376, January 2014. 